\title{
Microscopic structure of three-dimensional charge order in kagome superconductor AV3Sb5 and its tunability
}

\section{Mingu Kang}

Massachusetts Institute of Technology https://orcid.org/0000-0001-6991-0481

\section{Shiang Fang}

Massachusetts Institute of Technology https://orcid.org/0000-0002-9412-6426

Jonggyu Yoo

Max Planck POSTECH/Korea Research Initiative

\section{Brenden Ortiz}

University of California Santa Barbara

\section{Yuzki Oey}

University of California, Santa Barbara

\section{Sae Hee Ryu}

E. O. Lawrence Berkeley National Laboratory

\section{Jimin Kim}

Institute for Basic Science

\section{Chris Jozwiak}

Advanced Light Source, Lawrence Berkeley National Laboratory https://orcid.org/0000-0002-0980-

3753

\section{Aaron Bostwick}

Lawrence Berkeley National Laboratory https://orcid.org/0000-0002-9008-2980

\section{Eli Rotenberg}

E. O. Lawrence Berkeley National Laboratory https://orcid.org/0000-0002-3979-8844

\section{Efthimios Kaxiras}

Harvard University https://orcid.org/0000-0002-4682-0165

\section{Joseph Checkelsky}

Massachusetts Institute of Technology https://orcid.org/0000-0003-0325-5204

\section{Stephen Wilson}

University of California, Santa Barbara https://orcid.org/0000-0003-3733-930X

Jae-Hoon Park

Pohang University of Science and Technology https://orcid.org/0000-0001-6311-2454

Riccardo Comin ( $\nabla$ rcomin@mit.edu )

Masschusetts Institute of Technology 


\section{Research Article}

\section{Keywords:}

Posted Date: February 24th, 2022

DOI: https://doi.org/10.21203/rs.3.rs-1372848/v1

License: (c) (i) This work is licensed under a Creative Commons Attribution 4.0 International License. Read Full License

Version of Record: A version of this preprint was published at Nature Materials on November 3rd, 2022. See the published version at https://doi.org/10.1038/s41563-022-01375-2. 


\section{Microscopic structure of three-dimensional charge order in}

\section{kagome superconductor $A \mathrm{~V}_{3} \mathrm{Sb}_{5}$ and its tunability}

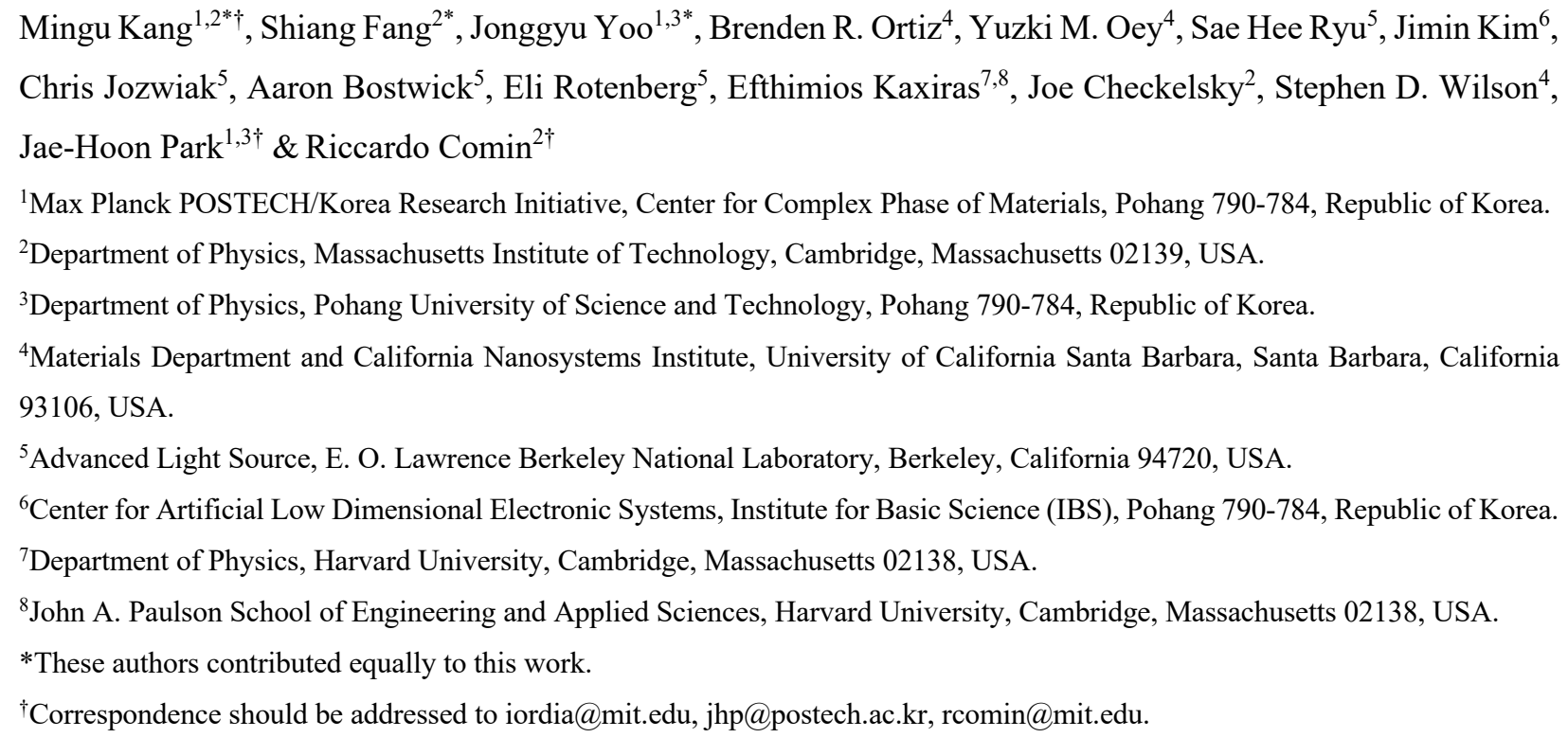

Correlated electronic systems are naturally susceptible to develop collective, symmetrybreaking electronic phases as observed in $\mathrm{Cu}$ - and Fe-based high-temperature superconductors, and twisted Moiré superlattices. The family of kagome metals $A V_{3} S_{5}(A$ $=\mathrm{K}, \mathrm{Rb}, \mathrm{Cs})$ is a recently discovered, rich platform to study many of these phenomena and their interplay. In these systems, three-dimensional charge order (3D-CO) is the primary instability that sets the stage in which other ordered phases emerge, including unidirectional stripe order, orbital flux order, and superconductivity. Therefore, determining the exact nature of the 3D-CO is key to capture the broader phenomenology in $A \mathrm{~V}_{3} \mathrm{Sb}_{5}$. Here, we use high-resolution angle-resolved photoemission spectroscopy to resolve the microscopic structure and symmetry of $3 \mathrm{D}-\mathrm{CO}$ in $A \mathrm{~V}_{3} \mathrm{Sb}_{5}$. Our approach is based on identifying an unusual splitting of kagome bands induced by 3D-CO, which provides a sensitive way to refine the spatial charge patterns in neighboring kagome planes. Notably, we found a marked dependence of the 3D-CO structure on alkali metal and doping: the 3D-CO in $\mathrm{CsV}_{3} \mathrm{Sb}_{5}$ is composed of kagome layers with alternating Star-of-David and Tri-Hexagonal distortions, while $\mathrm{KV}_{3} \mathrm{Sb}_{5}, \mathrm{RbV}_{3} \mathrm{Sb}_{5}$, and $\mathrm{Sn}$-doped $\mathrm{CsV}_{3} \mathrm{Sb}_{5}$ realize a staggered charge pattern breaking $C_{6}$ rotational symmetry. These results establish the microscopic structure of $3 \mathrm{D}-\mathrm{CO}$ and its evolution with chemical composition for the first time, providing fresh insights on the origin of the cascade of exotic electronic phases in $A \mathrm{~V}_{3} \mathrm{Sb}_{5}$. 
The family of $A \mathrm{~V}_{3} \mathrm{Sb}_{5}$ is a newly discovered series of kagome-based compounds realizing unconventional many-body phases and nontrivial electronic topology (Fig. 1c,d) ${ }^{1,2}$. In close analogy with other strongly correlated systems such as $\mathrm{Cu}$ - and Fe-based high-temperature superconductors $^{3,4}$, as well as twisted Moiré superlattices ${ }^{5}$, a cascade of coupled symmetry-broken electronic orders has been observed in $\mathrm{AV}_{3} \mathrm{Sb}_{5}$. These include translational symmetry breaking in the form of $2 \times 2$ charge order $(\mathrm{CO})$ below $T_{\mathrm{CO}} \approx 78 \sim 102 \mathrm{~K}^{2,6}$, rotational symmetry breaking in the form of unidirectional $1 \times 4$ stripe order below $T_{\mathrm{SO}} \approx 50 \sim 60 \mathrm{~K}^{7-10}$, a time-reversal symmetry breaking orbital flux phase below $T_{\mathrm{f}} \approx 70 \mathrm{~K}^{11}$, and superconductivity below $T_{\mathrm{c}} \approx 0.92 \sim 2.5 \mathrm{~K}^{2,12,13}$. Understanding the origin, nature, and interrelation between these electronic orders is a major frontier of this emerging research field.

Notably, previous theoretical and experimental investigations point toward the unique role of the electronic structure of the underlying kagome lattice in driving the rich physics of $A \mathrm{~V}_{3} \mathrm{Sb}_{5}{ }^{14}$ ${ }^{20}$. In the ideal limit, the kagome lattice exhibits multiple singularities in its electronic dispersion, including Dirac fermions at the Brillouin zone corner $K$, van Hove singularity (vHS) at the zone edge $M$, and flat bands across the whole Brillouin zone (Fig. 1a). Depending on the band filling fraction $n$, these electronic states may engender various topological and correlated phases as extensively investigated for more than a decade ${ }^{21-26}$. Especially at the vHS filling fractions $n=$ 3/12 and 5/12 (Fig. 1a), the Fermi surface of the kagome lattice is perfectly nested by three symmetry-equivalent reciprocal lattice vectors $Q_{1}=(0.5,0), Q_{2}$, and $Q_{3}$ (Fig. 1b). Combined with the high density of states at vHS, the nesting creates a diverging electronic susceptibility and sets the stage for pairing in multiple channels, and the subsequent emergence of charge/spin order and superconductivity ${ }^{24-26}$. The electronic structure of the $A \mathrm{~V}_{3} \mathrm{Sb}_{5}$ series follows this script, with multiple kagome-derived vHS sharply aligned to the Fermi level $\left(E_{F}\right)^{16-20}$. Accordingly, as displayed in Fig. 1e, density functional theory (DFT) calculation of phonon frequency reveals six unstable modes exactly at the $Q_{1}, Q_{2}$, and $Q_{3}$ in-plane wave vectors - three at $M\left(k_{z}=0\right)$ and the other three at $L\left(k_{z}=\pi\right)$ - indicating that the pristine kagome structure is unstable toward the $2 \times 2$ charge distortion ${ }^{27-29}$. Combined with the experimental identification of the $2 \times 2 \mathrm{CO}^{2,6}$, this suggests that the toy-model vHS physics of the ideal kagome lattice is indeed realized within the $A \mathrm{~V}_{3} \mathrm{Sb}_{5}$ system. 
67 dimensional kagome lattice. The three-dimensional nature of CO was reported in early studies ${ }^{17,30}$, supporting either twofold $(2 \times 2 \times 2)$ or fourfold $(2 \times 2 \times 4) c$-axis modulations ${ }^{31,32}$. As we illustrate in Fig. 1f,g, $M$-point ( $L$-point) phonons are associated to V-V bond distortions in-phase (out-of-phase) across neighboring kagome planes (Fig. 1f) ${ }^{28}$. Then, depending on the possible $3 Q$ combinations of $M$ and $L$ phonons, various microscopic $3 \mathrm{D}-\mathrm{CO}$ structures can be realized in the $A \mathrm{~V}_{3} \mathrm{Sb}_{5}$ series (Fig. 1h-m): Star-of-David or SoD $(-M,-M,-M)$; Tri-hexagonal or $\operatorname{TrH}(M, M, M)$; Alternating SoD and $\operatorname{TrH}(L, L, L)$; Staggered SoD $(-M,-L,-L)$; Staggered $\operatorname{TrH}(M, L, L)$; and Staggered Alternating SoD and $\operatorname{TrH}(M, M, L)$.

We emphasize here that identifying the exact structure and symmetry of 3D-CO is of paramount importance for understanding the sequence of electronic symmetry breaking transitions observed in $A \mathrm{~V}_{3} \mathrm{Sb}_{5}$. This is because $\mathrm{CO}$ formation has the highest energy scale in $A \mathrm{~V}_{3} \mathrm{Sb}_{5}$ series $\left(T_{\mathrm{CO}} \approx 78 \sim 102 \mathrm{~K}\right)$ and thus defines the background symmetry under which other electronic phases emerge (stripe order, nematicity, flux phase, and superconductivity). As an example, if $\mathrm{CO}$ crystallizes in the inverse $M L L, M L L$, or $M M L$ phases (Fig. $1 \mathrm{k}-\mathrm{m}), C_{6}$-rotational symmetry is spontaneously broken, which may provide a natural explanation for the unidirectional $1 \times 4$ stripe order and electronic nematicity emerging below $T_{\mathrm{CO}^{7}}{ }^{7-10}$. However, the detailed microscopic structure of $3 \mathrm{D}-\mathrm{CO}$ and its composition dependence in $A \mathrm{~V}_{3} \mathrm{Sb}_{5}$ series have not been conclusively reported so far, with different approaches - X-ray diffraction ${ }^{31,32}$, coherent phonon spectroscopy ${ }^{33}$, nuclear magnetic/quadrupole resonance ${ }^{34}$, scanning tunneling microscopy ${ }^{27}$, and $\mathrm{DFT}^{35}$ - yielding divergent results.

In the present study, we establish the microscopic structure of 3D-CO and its evolution in the $A \mathrm{~V}_{3} \mathrm{Sb}_{5}$ series by analyzing the detailed reconstruction of the electronic bands induced by 3DCO. Using high-resolution angle-resolved photoemission spectroscopy (ARPES), we observe an unusual energy splitting of the kagome-derived vHS and Dirac bands, which is a direct consequence of the unit cell reconstruction in the 3D-CO phase. Crucially, the precise morphology of the band splitting is highly sensitive to the intra-unit-cell stacking between different 3D-CO modulation patterns across adjacent kagome planes, which allows us to constrain the $3 \mathrm{D}-\mathrm{CO}$ structure and symmetry in the $A \mathrm{~V}_{3} \mathrm{Sb}_{5}$ series. Using this approach, we determine that the band splitting of $\mathrm{CsV}_{3} \mathrm{Sb}_{5}$ is most consistent with the Alternating $\mathrm{SoD}$ and $\mathrm{TrH}$ structure (LLL phase), while those in $\mathrm{KV}_{3} \mathrm{Sb}_{5}, \mathrm{RbV}_{3} \mathrm{Sb}_{5}$, and $\mathrm{Sn}$-doped $\mathrm{CsV}_{3} \mathrm{Sb}_{5}$ are markedly different from the $\mathrm{CsV}_{3} \mathrm{Sb}_{5}$ and can be assigned to the inverse $M L L$ or $M L L$ phases. These results expand our current 
understanding of $\mathrm{CO}$ in the $A \mathrm{~V}_{3} \mathrm{Sb}_{5}$ series, and reveal new essential details to explain the origin of multiple collective phenomena realized in strongly correlated kagome systems.

We start with a brief description of the overall electronic structure of $A \mathrm{~V}_{3} \mathrm{Sb}_{5}$. As displayed in Fig. 2a, the DFT band calculation for $\mathrm{CsV}_{3} \mathrm{Sb}_{5}$ reveals four bands near $\mathrm{E}_{\mathrm{F}}$ : an electron-pocket at the Brillouin zone center $\Gamma$ ( $G$-band), $d_{x y} / d_{x 2-y 2}$ orbital kagome bands with Dirac point at $\approx-0.27$ $\mathrm{eV}$ and vHS near $\mathrm{E}_{\mathrm{F}}\left(K l\right.$-band), $d_{x z} / d_{y z}$ orbital kagome bands with Dirac point at $\approx-1.3 \mathrm{eV}$ and vHS near $\mathrm{E}_{\mathrm{F}}\left(K 2\right.$-band), and additional $d_{x z} / d_{y z}$ orbital kagome bands with opposite parity from the $K 2$-band (K2'-band). All band dispersions have been closely reproduced in previous ARPES studies ${ }^{19,20,36-38}$. Meanwhile, we note that in the experimental geometry used in the present study, only $G$-, $K 1$-, and $K 2$-bands are visible in the ARPES spectra (see Fig. 2c for example) due to the destructive interference of photoelectrons from the $K 2^{2}$ '-band ${ }^{39}$. Additional characterization of the electronic structures in $A \mathrm{~V}_{3} \mathrm{Sb}_{5}$, such as Fermi surfaces and wide-range energy-momentum dispersions, can be found in the Extended Data Fig. 1.

As summarized in Fig. 2, we observed two distinct electronic reconstructions induced by $3 \mathrm{D}-\mathrm{CO}$ in $\mathrm{CsV}_{3} \mathrm{Sb}_{5}$. First, as shown in Fig. 2b-d, we detected clear shadow bands below $T_{\mathrm{CO}}$ (dashed arrows in Fig. 2d), which are the replica of the original bands (solid arrows in Fig. 2c,d) folded along the in-plane momentum direction. This is a direct consequence of the new periodicity arising from the in-plane component of charge order, which folds the pristine Brillouin zone to the smaller $2 \times 2$ CO Brillouin zone (see schematics in Fig. 2b). Such shadow bands and in-plane folding effects have been typically observed in other charge order systems such as transition metal dichalcogenides ${ }^{40}$ and rare-earth trichalcogenides ${ }^{41}$. At the same time, as shown in Fig. 2e-k, a detailed inspection below $T_{\mathrm{CO}}$ additionally reveals an unusual doubling or splitting of the kagome bands along the energy axis. Such splitting, which is unreported, could be visualized only after careful optimization of the spectral quality (see also Fig. 4f,g for corresponding energy and momentum distribution curves). At the simplest level, one can understand the band doubling as a consequence of the out-of-plane component of the 3D-CO, which folds the Brillouin zone along the $k_{z}$-direction and superimposes the $k_{z}=\pi \sim \pi / 2$ bands onto the $k_{z}=0 \sim \pi / 2$ bands (see schematics in Fig. 2e). In the case of $\mathrm{CsV}_{3} \mathrm{Sb}_{5}$, we find three sectors in the band structure where the doubling becomes most prominent: near the vHS of the Kl-band (Fig. 2g,k), at the lower Dirac band of the Kl-band (Fig. 2g,i), and at the K2-band near $\mathrm{E}_{\mathrm{F}}$ (Fig. 2k). 
The key idea of our study comes from the recognition that the doubled-band dispersion in the 3D-CO state is actually more than the simple superposition of $k_{z}=0 \sim \pi / 2$ and $k_{z}=\pi \sim \pi / 2$ bands of the pristine structure. In the $3 \mathrm{D}-\mathrm{CO}$ state, the adjacent kagome layers in $A \mathrm{~V}_{3} \mathrm{Sb}_{5}$ become distinct upon realizing different $\mathrm{CO}$ patterns on each layer (Fig. $1 \mathrm{j}-\mathrm{m}$ ). The altered hopping pathways between the two charge-ordered kagome planes further reconstruct the doubled-band dispersion. This mechanism depends on the detailed charge distortions in adjacent kagome planes, with the nature of the band splitting becoming strongly dependent on the 3D-CO structure. This provides a unique and highly constrained way to resolve the microscopic structure of $3 \mathrm{D}-\mathrm{CO}$ in $A \mathrm{~V}_{3} \mathrm{Sb}_{5}$ series.

To illustrate this idea further, we simulated the reconstruction of $\mathrm{CsV}_{3} \mathrm{Sb}_{5}$ bands in all 138 possible 3D-CO structures using DFT. Fig. 3a-f represents the electronic structure of $\mathrm{CsV}_{3} \mathrm{Sb}_{5}$ at $k_{z}=0$ in the inverse $M M M, M M M, L L L$, inverse $M L L, M L L$, and $M M L$ phases, respectively. Note that, for simplicity, we unfolded the band structure along the in-plane momentum-space, while keeping the band folding along $k_{z}$. In accordance with the experimental results, the doubling of the dispersions for the $K 1$ - and $K 2$-kagome bands are closely reproduced in the 3D-CO states (see yellow arrows in Fig. 3c for example). Most importantly, the details of band splitting are highly dependent on the microscopic structure of 3D-CO. The most noticeable discriminant between different 3D-CO structures is the behavior of the lower K1-Dirac band. As marked with yellow arrows in the insets of Fig. 3, the lower $K 1$-Dirac band barely splits in the inverse $M M M, M M M$, inverse $M L L$, and $M L L$ structures (Fig. 3a,b,d,e), while an apparent doubling is observed in the $L L L$ structure (Fig. 3c). The latter closely reproduces the ARPES spectra in Fig. 2g,i. For the case of the $M M L$ structure (Fig. 3f), both the lower K1-Dirac band and K1-vHS band split into at least three bands if one takes an average over all possible $C_{2}$-symmetric domains (see also the Extended Data Fig. 2 for the domain-resolved calculations). Based on the above considerations, we conclude that the observed band splitting supports the $L L L$ structure or Alternating SoD and TrH phase (Fig. $1 \mathrm{j})$ as the microscopic 3D-CO structure in $\mathrm{CsV}_{3} \mathrm{Sb}_{5}$.

Intriguingly, the investigation of $\mathrm{KV}_{3} \mathrm{Sb}_{5}, \mathrm{RbV}_{3} \mathrm{Sb}_{5}$, and $\mathrm{Sn}$-doped $\mathrm{CsV}_{3} \mathrm{Sb}_{5}$ revealed an electronic reconstruction markedly different from the $\mathrm{CsV}_{3} \mathrm{Sb}_{5}$ case. Figure 4 displays ARPES spectra of $\mathrm{KV}_{3} \mathrm{Sb}_{5}, \mathrm{RbV}_{3} \mathrm{Sb}_{5}$, and $\mathrm{Sn}$-doped $\mathrm{CsV}_{3} \mathrm{Sb}_{5}$ measured at $6 \mathrm{~K}$, in the $\mathrm{CO}$ state (See Extended Data Fig. 3 for the transport characterization of superconductivity and CO in Sn-doped and K2-bands (Fig. 4d,e) is clearly observed across the whole family. The corresponding energy 
distribution curves of the $K 1$-vHS and momentum-distribution curves of the K2-band (Fig. 4f,g) also unambiguously demonstrate the presence of band doubling. These results indicate that the 3D-CO is a universal phenomenon in the $A \mathrm{~V}_{3} \mathrm{Sb}_{5}$ series. However, we observe that the behavior of the lower $\mathrm{K} 1$ Dirac band in $\mathrm{KV}_{3} \mathrm{Sb}_{5}, \mathrm{RbV}_{3} \mathrm{Sb}_{5}$, and $\mathrm{Sn}$-doped $\mathrm{CsV}_{3} \mathrm{Sb}_{5}$ is very different from that of the $\mathrm{CsV}_{3} \mathrm{Sb}_{5}$ case. As highlighted with yellow arrows in Fig. 4a-e, the lower Dirac dispersion of the $K l$ band does not undergo a splitting in the $3 \mathrm{D}-\mathrm{CO}$ state, in contrast to the observations in $\mathrm{CsV}_{3} \mathrm{Sb}_{5}$ (Fig. 2g,i). Compared to the calculations in Fig. 3, this behavior rules out the $L L L$ and $M M L$ structures (Fig. 3c,f) but is consistent with the inverse $M M M, M M M$, inverse $M L L$, and $M L L$ structures (Fig. 3a,b,d,e). In case of the inverse $M M M$ and $M M M$ structures however, the charge distortions do not distinguish the neighboring kagome planes (i.e. $2 \times 2 \times 1$ structure) and cannot induce the out-of-plane band doubling observed on the $K 1$-vHS and $K 2$-band. We thus conclude that the $3 \mathrm{D}-\mathrm{CO}$ in $\mathrm{KV}_{3} \mathrm{Sb}_{5}, \mathrm{RbV}_{3} \mathrm{Sb}_{5}$, and $\mathrm{Sn}$-doped $\mathrm{CsV}_{3} \mathrm{Sb}_{5}$ manifests in the inverse $M L L$ or $M L L$ phases (intrinsically breaking $C_{6}$-rotational symmetry) at variance with the $L L L$ structure in pristine $\mathrm{CsV}_{3} \mathrm{Sb}_{5}$. This implies that despite the charge ordering tendency of the kagome lattice is universal in $A \mathrm{~V}_{3} \mathrm{Sb}_{5}$ family, the microscopic details of 3D-CO are strongly dependent on the chemical composition and doping, adding to the rich physics that can be realized in the $A \mathrm{~V}_{3} \mathrm{Sb}_{5}$ series.

Lastly, we discuss the insights obtained from our results in relation to the existing literature. Previous DFT calculations have revealed that the net energy of $A \mathrm{~V}_{3} \mathrm{Sb}_{5}$ only marginally depends on the out-of-plane structure of 3D-CO, indicating that the charge order is only weakly correlated along the $c$-axis ${ }^{35}$. It is thus quite possible that the detailed microscopic structure of 3D-CO varies sensitively depending on the interlayer spacing or strength of the inter-kagome-layer interactions, which can be controlled by the alkali metal element ${ }^{1}$ or Sn-doping to Sb1 sites ${ }^{42}$. Intriguingly, several DFT studies have predicted the MLL structure or Staggered $\mathrm{TrH}$ phase to be the ground state of $A \mathrm{~V}_{3} \mathrm{Sb}_{5}{ }^{29,33}$, consistent with our conclusions for $\mathrm{KV}_{3} \mathrm{Sb}_{5}, \mathrm{RbV}_{3} \mathrm{Sb}_{5}$, and $\mathrm{Sn}$-doped $\mathrm{CsV}_{3} \mathrm{Sb}_{5}$. In the case of pristine $\mathrm{CsV}_{3} \mathrm{Sb}_{5}$, it is still being debated whether the $3 \mathrm{D}-\mathrm{CO}$ manifests in the $2 \times 2 \times 2$ form as in the $\mathrm{K}$ and $\mathrm{Rb}$ counterparts, or in a more complicated $2 \times 2 \times 4$ pattern $^{31,32}$. Nevertheless, the X-ray structural refinements on $\mathrm{CsV}_{3} \mathrm{Sb}_{5}$ revealed the modulation pattern containing an alternating $\mathrm{TrH}$ and SoD structure without staggering ${ }^{31}$, in close analogy with the $L L L$ structure obtained here. We note that in $\mathrm{CsV}_{3} \mathrm{Sb}_{5}$, signatures of bulk rotational symmetry breaking and surface stripe order emerge only below the characteristic temperature $T_{\mathrm{SO}} \approx 60 \mathrm{~K}$ and not in the 
pure charge-ordered state between $T_{\mathrm{CO}} \approx 94 \mathrm{~K}$ and $T_{\mathrm{SO}} \approx 60 \mathrm{~K}^{7,10}$. This suggests that the $3 \mathrm{D}-\mathrm{CO}$

191 itself does not break $C_{6}$ rotational symmetry, which is indeed the case for the $L L L$ structure 192 revealed in the present study (Fig. 1j). The nematicity and surface stripe order observed in $\mathrm{CsV}_{3} \mathrm{Sb}_{5}$ 193 thus represent additional emergent electronic symmetry breaking under the 3D-CO state. In 194 contrast, we conclude that the $C_{6}$ rotational symmetry to be already broken in the 3D-CO phase of $195(\mathrm{~K}, \mathrm{Rb}) \mathrm{V}_{3} \mathrm{Sb}_{5}$ with the $M L L$ or inverse $M L L$ structure (Fig. 1k,1). Indeed, the Fourier-transformed 196 scanning tunneling spectroscopy on $\mathrm{KV}_{3} \mathrm{Sb}_{5}$ revealed that the symmetry of system is reduced to $197 C_{2}$ in the charge-ordered state even without stripe ordering ${ }^{9}$. However, the phenomena of 3D-CO 198 and rotational symmetry breaking are much less explored in $(\mathrm{K}, \mathrm{Rb}) \mathrm{V}_{3} \mathrm{Sb}_{5}$ compared to the Cs case 199 - the present results thus invite future X-ray diffraction, nuclear magnetic resonances, and 200 transport investigations on the full series.

201 In conclusion, we establish the microscopic structure of 3D-CO and its tunability in the 202 family of topological kagome metals $A \mathrm{~V}_{3}(\mathrm{Sb}, \mathrm{Sn})_{5}$. Determining the exact structure of $3 \mathrm{D}-\mathrm{CO}$ is a 203 topic of great relevance, as it sets the background symmetry of the system under which other many204 body effects emerge. Combining high-resolution ARPES and DFT supercell calculations, we 205 resolve the detailed electronic reconstruction of the kagome bands in the 3D-CO state. We reveal 206 that the 3D-CO in $\mathrm{CsV}_{3} \mathrm{Sb}_{5}$ consists of a stacking of kagome layers with alternating SoD and $\mathrm{TrH}$ 207 distortions $(L L L)$, while $\mathrm{KV}_{3} \mathrm{Sb}_{5}, \mathrm{RbV}_{3} \mathrm{Sb}_{5}$, and $\mathrm{Sn}$-doped $\mathrm{CsV}_{3} \mathrm{Sb}_{5}$ realize the staggered $\mathrm{CO}$ 208 structure breaking the $C_{6}$ rotational symmetry ( $M L L$ or inverse $\left.M L L\right)$. The remarkable tunability 209 of the $3 \mathrm{D}-\mathrm{CO}$ state across otherwise similar compounds suggests that the $A \mathrm{~V}_{3} \mathrm{Sb}_{5}$ series is a 210 candidate host for an extremely rich phase diagram of emergent electronic phases, enabling new 211 opportunities for fundamental studies at the nexus of strong correlation phenomena and topology. 


\section{Methods}

\section{Sample synthesis and angle-resolved photoemission spectroscopy}

High-quality single crystals of pristine and $\mathrm{Sn}$-doped $A \mathrm{~V}_{3} \mathrm{Sb}_{5}$ were synthesized via flux method as described in Ref. ${ }^{1,2,42}$. ARPES experiments were performed at Beamline 7.0.2 216 (MAESTRO) of the Advanced Light Source, equipped with R4000 hemispherical electron

217 analyzer (Scienta Omicron). The samples were cleaved inside an ultrahigh vacuum chamber with 218 a base pressure better than $\approx 4 \times 10^{-11}$ torr. We keep the following experimental geometry 219 throughout the measurement: horizontal analyzer slit, linear horizontal light polarization, and $\Gamma$ 220 K-M direction of the sample aligned to the scattering plane. For each sample, photon energy was 221 scanned from $60 \mathrm{eV}$ to $200 \mathrm{eV}$, covering more than three complete three-dimensional Brillouin 222 zone. For the high-resolution data in Fig. 2,4, we selected the photon energies for each sample that 223 best visualize the band splitting. The energy and momentum resolutions were better than $20 \mathrm{meV}$ 224 and $0.01 \AA^{-1}$.

\section{Density functional theory calculations}

DFT calculations were performed using the Vienna Ab initio Simulation Package ${ }^{43,44}$, with GGA-PBE exchange-correlation functional ${ }^{45}$ and the pseudo potential formalism based on the Projector Augmented Wave method ${ }^{46}$. The phonon frequency spectrum was derived from the Hessian matrix (which encodes the second derivatives of the atomic position coordinate) computed with the density-functional-perturbation theory (DFPT) method, using a $4 \times 4 \times 2$ supercell unit ${ }^{47}$.

232 We have further simulated various $2 \times 2 \times 2 \mathrm{CO}$ states from combinations of the unstable $M$ and $L$ 233 phonon modes. The CO structures were relaxed with a $350 \mathrm{eV}$ energy cutoff for the plane-wave 234 basis and a $4 \times 4 \times 2$ grid sampling in the momentum space Brillouin zone. To elucidate the electronic properties of these COs, we performed electronic band structure unfolding and projections based on the Wannier models derived from DFT ground states, using Wannier 90 code. 


\section{Acknowledgements}

238 This work was supported by the Air Force Office of Scientific Research Young Investigator 239 Program under grant FA9550-19-1-0063, and by the STC Center for Integrated Quantum Materials 240 (NSF grant no. DMR-1231319). The work is funded in part by the Gordon and Betty Moore 241 Foundation's EPiQS Initiative, Grant GBMF9070 to JGC. The works at Max Planck 242 POSTECH/Korea Research Initiative were supported by the National Research Foundation of 243 Korea, Ministry of Science, Grant No. 2016K1A4A4A01922028 and 2020M3H4A2084417. 244 B.R.O. and S.D.W. were supported by the National Science Foundation (NSF) through Enabling 245 Quantum Leap: Convergent Accelerated Discovery Foundries for Quantum Materials Science, 246 Engineering and Information (Q-AMASE-i): Quantum Foundry at UC Santa Barbara (DMR247 1906325). This research used resources of the Advanced Light Source, a U.S. DOE Office of 248 Science User Facility under contract no. DE-AC02-05CH11231. M.K. acknowledges a Samsung 249 Scholarship from the Samsung Foundation of Culture. B.R.O. acknowledges support from the 250 California NanoSystems Institute through the Elings Fellowship program.

\section{Author contributions}

253 M.K., J.-H.P, and R.C. conceived the project; M.K. and J.Y. performed the ARPES experiments 254 and analyzed the resulting data with help from S.H.R., J.K., C.J., A.B., and E.L.; S.F. performed 255 the theoretical calculations with help from E.K., J.C.; B.R.O., Y.M.O., and S.D.W. synthesized 256 and characterized the crystals. M.K. and R.C. wrote the manuscript with input from all coauthors.

\section{Data availability}

259 The datasets presented within this study are available from the corresponding authors upon 260 reasonable request.

\section{Competing interests}

263 The authors declare no competing interests 


\section{References}

265 1. Ortiz, B. R. et al. New kagome prototype materials: Discovery of KV3Sb5,RbV3Sb5, and

2. Ortiz, B. R. et al. CsV3Sb5: a Z2 topological kagome metal with a superconducting ground state. Phys. Rev. Lett. 125, 247002 (2020).

3. Keimer, B., Kivelson, S. A., Norman, M. R., Uchida, S. \& Zaanen, J. From quantum matter to high-temperature superconductivity in copper oxides. Nature 518, 179-186 (2015).

4. Si, Q., Yu, R. \& Abrahams, E. High-temperature superconductivity in iron pnictides and chalcogenides. Nat. Rev. Mater. 1, 1 (2016).

5. Cao, Y. et al. Unconventional superconductivity in magic-angle graphene superlattices. Nature 556, 43-50 (2018).

6. Jiang, Y.-X. et al. Unconventional chiral charge order in kagome superconductor KV3Sb5. Nat. Mater. 20, 1353-1357 (2021).

7. Zhao, H. et al. Cascade of correlated electron states in a kagome superconductor CsV3Sb5. Nature 599, 216-221 (2021).

8. Shumiya, N. et al. Intrinsic nature of chiral charge order in the kagome superconductor $\mathrm{Rb}$ V3Sb5. Phys. Rev. B 104, 035131 (2021).

9. Li, H. et al. Rotation symmetry breaking in the normal state of a kagome superconductor KV3Sb5. Nat. Phys. (2022) doi:10.1038/s41567-021-01479-7.

10. Xiang, Y. et al. Twofold symmetry of c-axis resistivity in topological kagome superconductor CsV3Sb5 with in-plane rotating magnetic field. Nat. Commun. 12, 6727 (2021).

11. $\mathrm{Yu}, \mathrm{L}$. et al. Evidence of a hidden flux phase in the topological kagome metal CsV\$3\$Sb\$_5.arXiv 2107.10714 (2021).

12. Ortiz, B. R. et al. Superconductivity in the Z2 kagome metal KV3Sb5. Phys. Rev. Mat. 5, 034801 (2021).

13. Yin, Q. et al. Superconductivity and normal-state properties of kagome metal RbV3Sb5 single crystals. Chinese Phys. Lett. 38, 037403 (2021).

14. Wu, X. et al. Nature of Unconventional Pairing in the Kagome Superconductors A V $3 \mathrm{Sb}$ 5 ( A = K, Rb, Cs ). Phys. Rev. Lett. 127, 177001 (2021).

15. Tazai, R., Yamakawa, Y., Onari, S. \& Kontani, H. Mechanism of exotic density-wave and beyond-Migdal unconventional superconductivity in kagome metal AV $3 \mathrm{Sb} 5(\mathrm{~A}=\mathrm{K}, \mathrm{Rb}$, Cs). Arxiv 2107.05372 (2021).

16. Zhou, X. et al. Origin of charge density wave in the kagome metal CsV3 Sb5 as revealed by optical spectroscopy. Phys. Rev. B 104, L041101 (2021).

17. Li, H. et al. Observation of Unconventional Charge Density Wave without Acoustic Phonon Anomaly in Kagome Superconductors A V3Sb5 (A=Rb, Cs). Phys. Rev. X11, 031050 (2021).

18. Nakayama, K. et al. Multiple energy scales and anisotropic energy gap in the chargedensity-wave phase of the kagome superconductor CsV 3 Sb 5. Phys. Rev. B 104, L161112 (2021).

19. Liu, Z. et al. Charge-Density-Wave-Induced Bands Renormalization and Energy Gaps in a Kagome Superconductor RbV 3 Sb 5 . Phys. Rev. X 11, 41010 (2021).

20. Kang, M. et al. Twofold van Hove singularity and origin of charge order in topological kagome superconductor CsV3Sb5. Nat. Phys. (2022) doi:10.1038/s41567-021-01451-5. 
21. Guo, H. M. \& Franz, M. Topological insulator on the kagome lattice. Phys. Rev. B 80, 113102 (2009).

22. $\mathrm{Xu}, \mathrm{G}$. , Lian, B. \& Zhang, S.-C. Intrinsic Quantum Anomalous Hall Effect in the Kagome Lattice Cs2LiMn3F12. Phys. Rev. Lett. 115, 186802 (2015).

23. Tang, E., Mei, J.-W. \& Wen, X.-G. High-Temperature Fractional Quantum Hall States. Phys. Rev. Lett. 106, 236802 (2011).

24. Yu, S. L. \& Li, J. X. Chiral superconducting phase and chiral spin-density-wave phase in a Hubbard model on the kagome lattice. Phys. Rev. B 85, 4-7 (2012).

25. Kiesel, M. L., Platt, C. \& Thomale, R. Unconventional fermi surface instabilities in the kagome hubbard model. Phys. Rev. Lett. 110, 126405 (2013).

26. Wang, W., Li, Z., Xiang, Y. \& Wang, Q. Competing electronic orders on kagome lattices at van Hove filling. Phys. Rev. B 87, 115135 (2013).

27. Tan, H., Liu, Y., Wang, Z. \& Yan, B. Charge density waves and electronic properties of superconducting kagome metals. Phys. Rev. Lett. 127, 046401 (2021).

28. Christensen, M. H., Birol, T., Andersen, B. M. \& Fernandes, R. M. Theory of the charge density wave in AV3Sb5 kagome metals. Phys. Rev. B 104, 214513 (2021).

29. Subedi, A. Hexagonal-to-base-centered-orthorhombic 4Q charge density wave order in kagome metals KV3Sb5, RbV3Sb5, and CsV3Sb5. Phys. Rev. Mater. 6, 015001 (2022).

30. Liang, Z. et al. Three-Dimensional Charge Density Wave and Surface-Dependent VortexCore States in a Kagome Superconductor CsV3Sb5. Phys. Rev. X 11, 31026 (2021).

31. Ortiz, B. R. et al. Fermi Surface Mapping and the Nature of Charge-Density-Wave Order in the Kagome Superconductor CsV3Sb5. Phys. Rev. X11, 41030 (2021).

32. Li, H. et al. Spatial symmetry constraint of charge-ordered kagome superconductor.

33. Ratcliff, N., Hallett, L., Ortiz, B. R., Wilson, S. D. \& Harter, J. W. Coherent phonon spectroscopy and interlayer modulation of charge density wave order in the kagome metal CsV3Sb5. Phys. Rev. Mater. 5, L111801 (2021).

34. Luo, J. et al. Star-of-David pattern charge density wave with additional modulation in the kagome superconductor CsV\$_3\$Sb\$_5 revealed by $\$^{\wedge}\{51\} \$ V-N M R$ and $\$^{\wedge}\{121 / 123\} \$ S b-N Q R$. arXiv 2108.10263 (2021).

35. Ye, Z., Luo, A., Yin, J.-X., Hasan, M. Z. \& Xu, G. Structural instability and charge modulations in the Kagome superconductor \$A\$V\$_3Sb\$5\$. arXiv 2111.07314 (2021).

36. Hu, Y. et al. charge order assisted topological surface states and flat bands in the kagome superconductor CsV3Sb5. Sci. Bull. (2022).

37. Luo, H. et al. Electronic Nature of Charge Density Wave and Electron-Phonon Coupling in Kagome Superconductor KV\$_3\$Sb\$_5. Nat. Commun. 13, 273 (2022).

38. Cho, S. et al. Emergence of new van Hove singularities in the charge density wave state of a topological kagome metal RbV3Sb5. Phys. Rev. Lett. 127, 236401 (2021).

39. $\mathrm{Hu}, \mathrm{Y}$. Rich Nature of Van Hove Singularities in Kagome Superconductor CsV3Sb5 Yong. arXiv 2106.05922 (2021).

40. Rossnagel, K. On the origin of charge-density waves in select layered transition-metal dichalcogenides. J. Phys. Condens. Matter 23, (2011).

41. Brouet, V. et al. Angle-resolved photoemission study of the evolution of band structure and charge density wave properties in RTe3. Phys. Rev. B 77, 235104 (2008).

42. Oey, Y. M. et al. Fermi level tuning and double-dome superconductivity in the kagome metals CsV\$_3\$Sb\$_\{5-x\}\$Sn\$_x\$. arXiv 2110.10912 (2021).

43. Kresse, G. \& Furthmüller, J. Efficiency of ab-initio total energy calculations for metals 
and semiconductors using a plane-wave basis set. Comput. Mater. Sci. 6, 15-50 (1996).

44. G. Kresse \& Furthmu" ller, J. Efficient iterative schemes for ab initio total-energy calculations using a plane-wave basis set. Phys. Rev. B 54, 11169-11186 (1996).

45. Perdew, J. P., Burke, K. \& Ernzerhof, M. Generalized gradient approximation made simple. Phys. Rev. Lett. 77, 3865-3868 (1996).

46. Blochl, P. E. Projector augmented-wave method. Phys. Rev. B 50, 17953-17979 (1994).

47. Togo, A. \& Tanaka, I. First principles phonon calculations in materials science. Scr. Mater. 108, 1-5 (2015). 

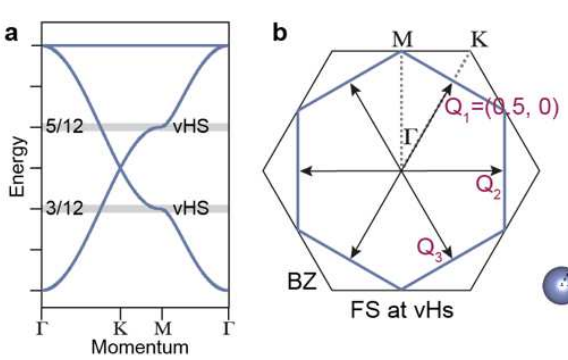

c

Top view
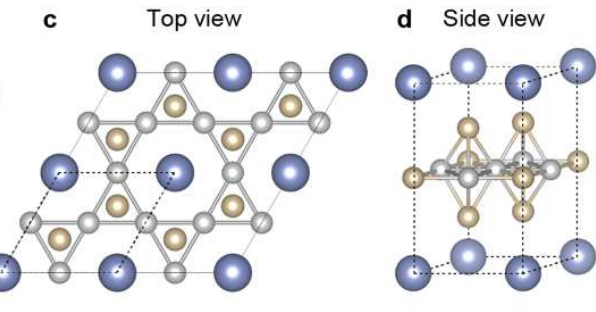

Unstable phonon modes

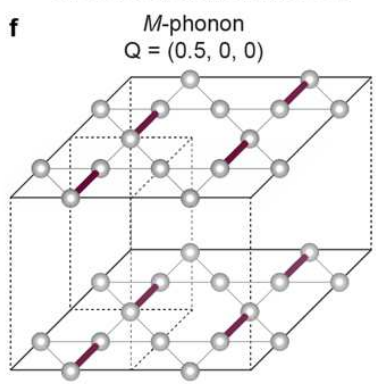

g

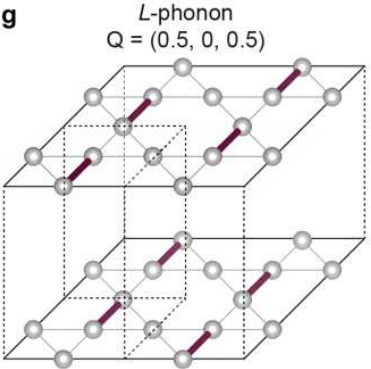

h

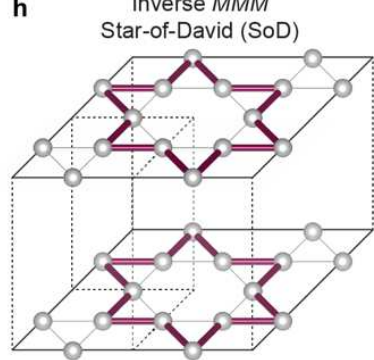

k

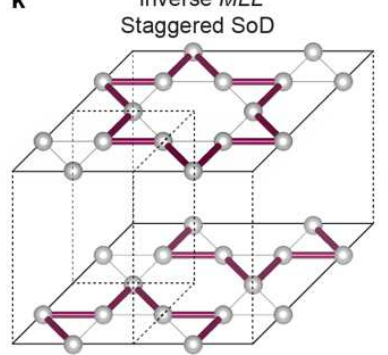

Possible 3Q-CDW orders

i

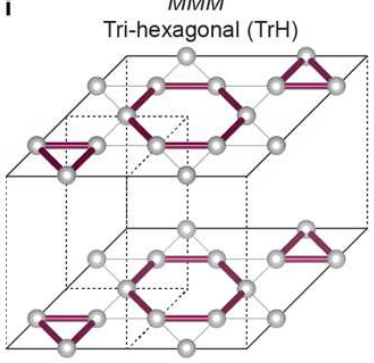

I

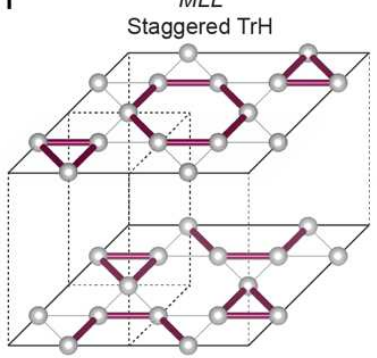

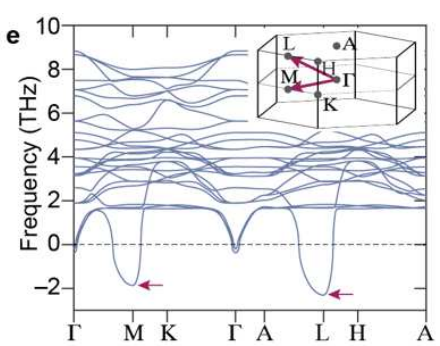

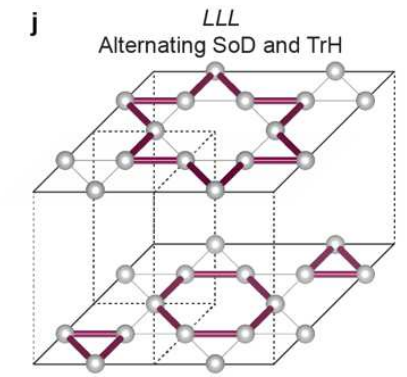

m

Staggered Alternating SoD and TrH

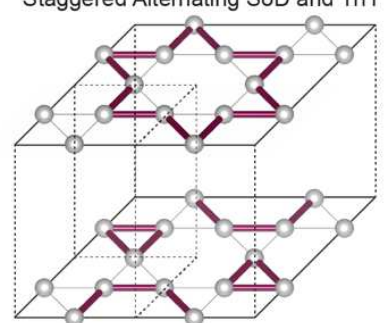

Figure 1 | Possible microscopic structures of the three-dimensional charge order (3D-CO) in

kagome metal $\boldsymbol{A} \mathbf{V}_{3} \mathrm{Sb}_{5}$. a, Tight-binding electronic structure of an ideal kagome lattice. Greyshaded lines mark the vHS filling fractions at 3/12 and 5/12. b, Perfectly nested hexagonal Fermi surface of the kagome lattice at the vHS filling fractions. Double-headed arrows indicate three symmetry-equivalent nesting vectors $Q_{1}, Q_{2}$, and $Q_{3}$. c,d, Crystal structure of the $A \mathrm{~V}_{3} \mathrm{Sb}_{5}$ with a V-kagome net. Dashed lines mark the unit cell in the undistorted phase. e, Calculated phonon

371 dispersion of $\mathrm{CsV}_{3} \mathrm{Sb}_{5}$ showing instabilities of the pristine structure at $M$ and $L$. Inset shows the

372 Brillouin zone. f,g, Lattice distortions corresponding to the instabilities at $M$ and $L$ phonons, respectively. h-m, Possible structures of the $3 \mathrm{D}-\mathrm{CO}$ in $A \mathrm{~V}_{3} \mathrm{Sb}_{5}$ based on $3 Q$-combinations of the $M$ and $L$ phonons. 

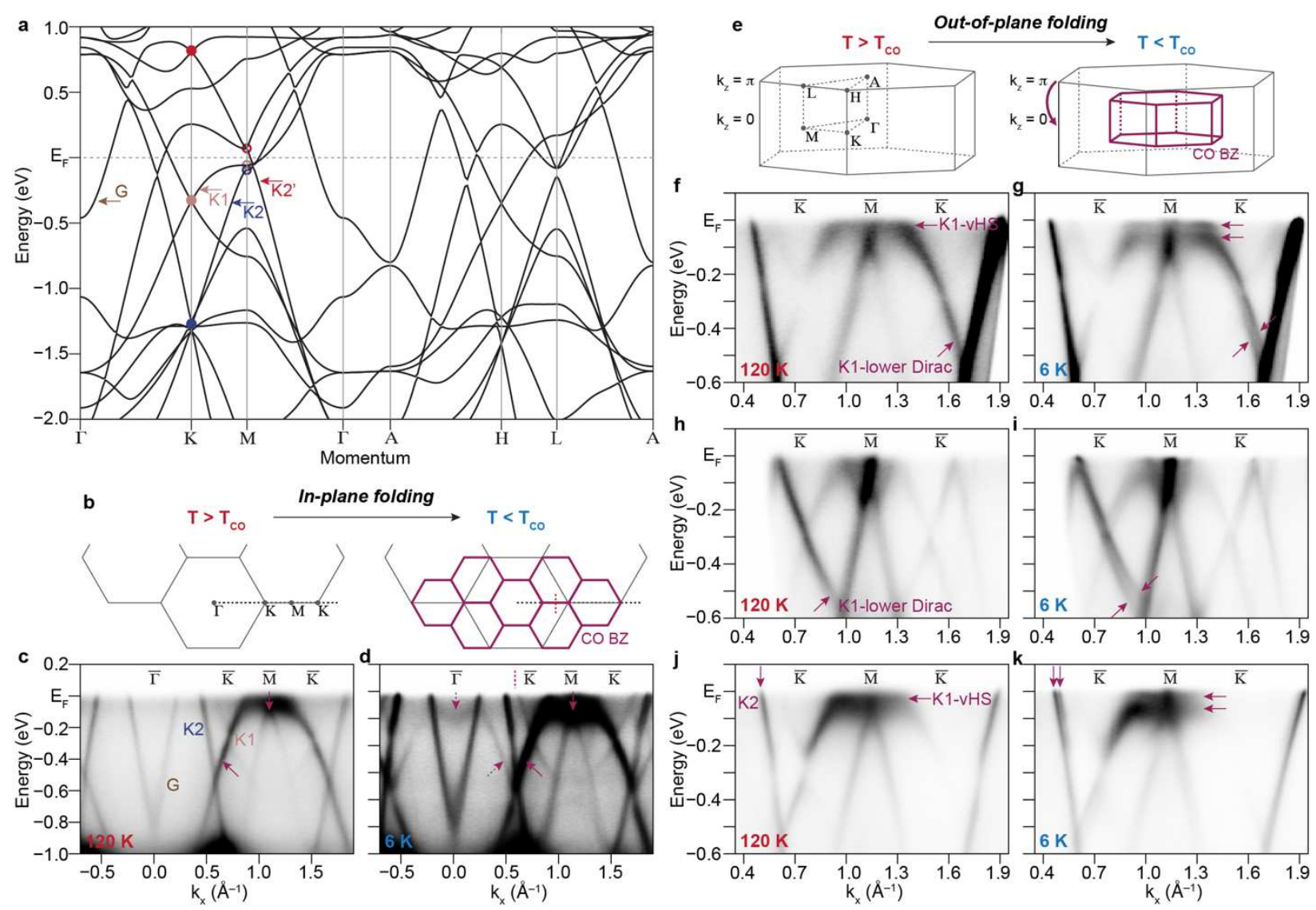

Figure 2 Two distinct types of electronic reconstructions in $\mathrm{CsV}_{3} \mathrm{Sb}_{5}$ induced by 3D-CO. a,

DFT band structure of $\mathrm{CsV}_{3} \mathrm{Sb}_{5}$ showing four bands crossing the Fermi level: $G, K 1, K 2$, and $K 2$ 'bands. The Dirac points at $K$ and vHS at $M$ emerging from the $K 1, K 2$, and $K 2$ ' kagome bands are marked with filled and open circles, respectively. b-d, Electronic reconstruction from the in-plane component of charge order. b, Schematics of the in-plane folding of the Brillouin zone. c,d, Experimental band dispersion of $\mathrm{CsV}_{3} \mathrm{Sb}_{5}$ measured at $120 \mathrm{~K}$ and $6 \mathrm{~K}$ (above and below $\mathrm{T}_{\mathrm{CO}}$ ), respectively. Solid arrows in c,d mark the original bands, while the dashed arrows in $\mathrm{d}$ indicate the replica bands. e-k, Electronic reconstruction from the out-of-plane component of 3D-CO. e, Schematics of the out-of-plane folding of the Brillouin zone. Panels $f, h, j$ (g,i,k) represent the dispersions measured above (below) $\mathrm{T}_{\mathrm{CO}}$, at the first Brillouin zone with photon energy $86 \mathrm{eV}$, at the second Brillouin zone with photon energy $86 \mathrm{eV}$, and at the first Brillouin zone with photon energy $113 \mathrm{eV}$, respectively. Solid arrows in g,i,k indicate the doubling or splitting of the kagome bands in the 3D-CO state. 

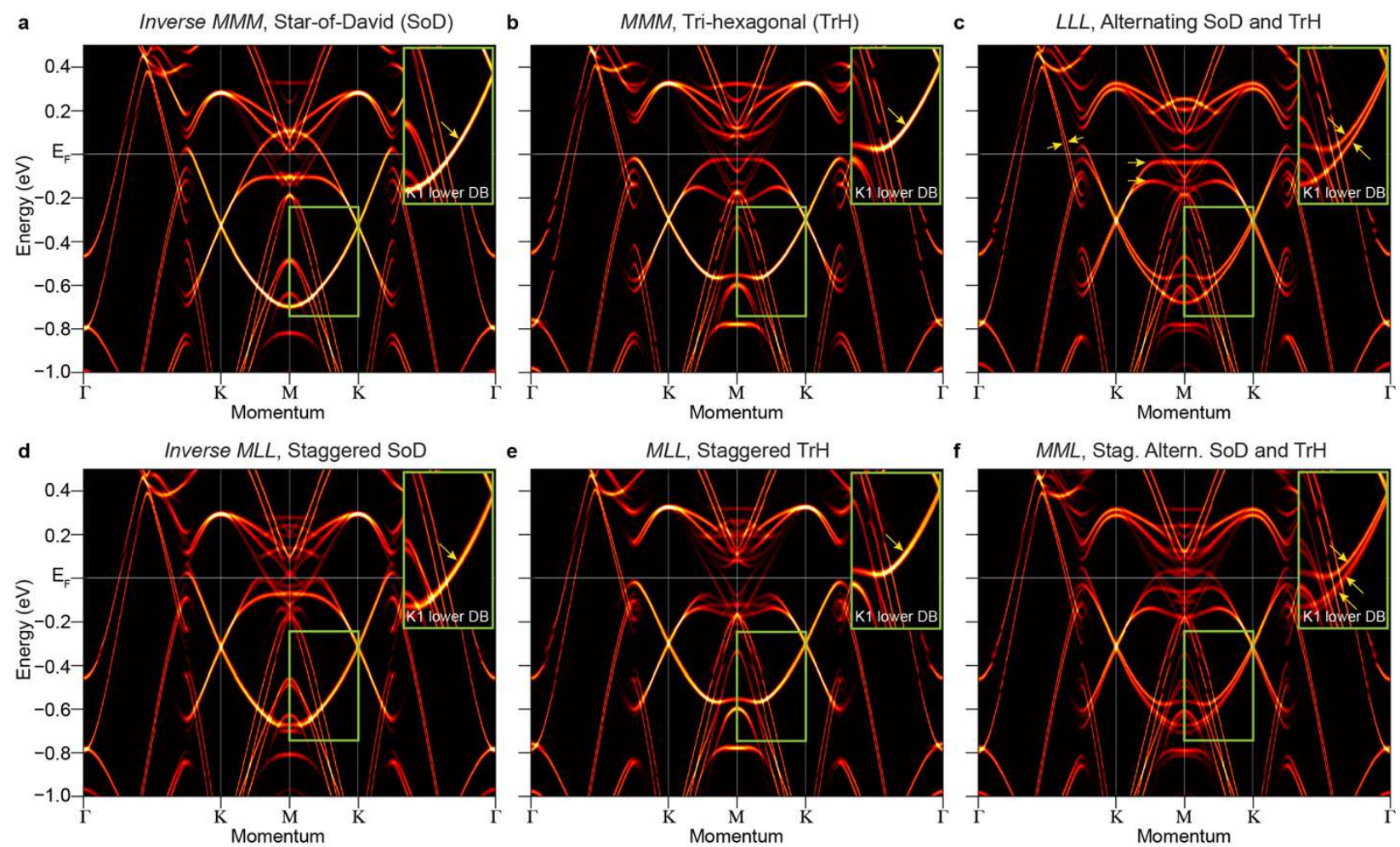

Figure 3 | Theoretical calculation of electronic reconstruction in $A \mathrm{~V}_{3} \mathrm{Sb}_{5}$ and its dependence on the microscopic structure of 3D-CO. a-f, Calculated dispersion of $\mathrm{CsV}_{3} \mathrm{Sb}_{5}$ at $k_{z}=0$ in the inverse $M M M, M M M, L L L$, inverse $M L L, M L L$, and $M M L$ phases, respectively. For simplicity, the

391 dispersion is unfolded along the in-plane momentum direction. Yellow arrows in c highlight the 392 splitting of $K 1$ and $K 2$ bands in the 3D-CO state. For the inverse $M L L, M L L$ and $M M L$ phases (d393 f) the calculation is averaged over three $C_{2}$ symmetric charge order domains to account for the 394 macroscopic beam spot size. Corresponding domain-resolved dispersions are presented in the 395 Extended Data Fig. 2. The insets zoom in the behavior of the lower K1 Dirac band, whose splitting 396 sensitively depends on the microscopic structure of 3D-CO (see yellow arrows). 

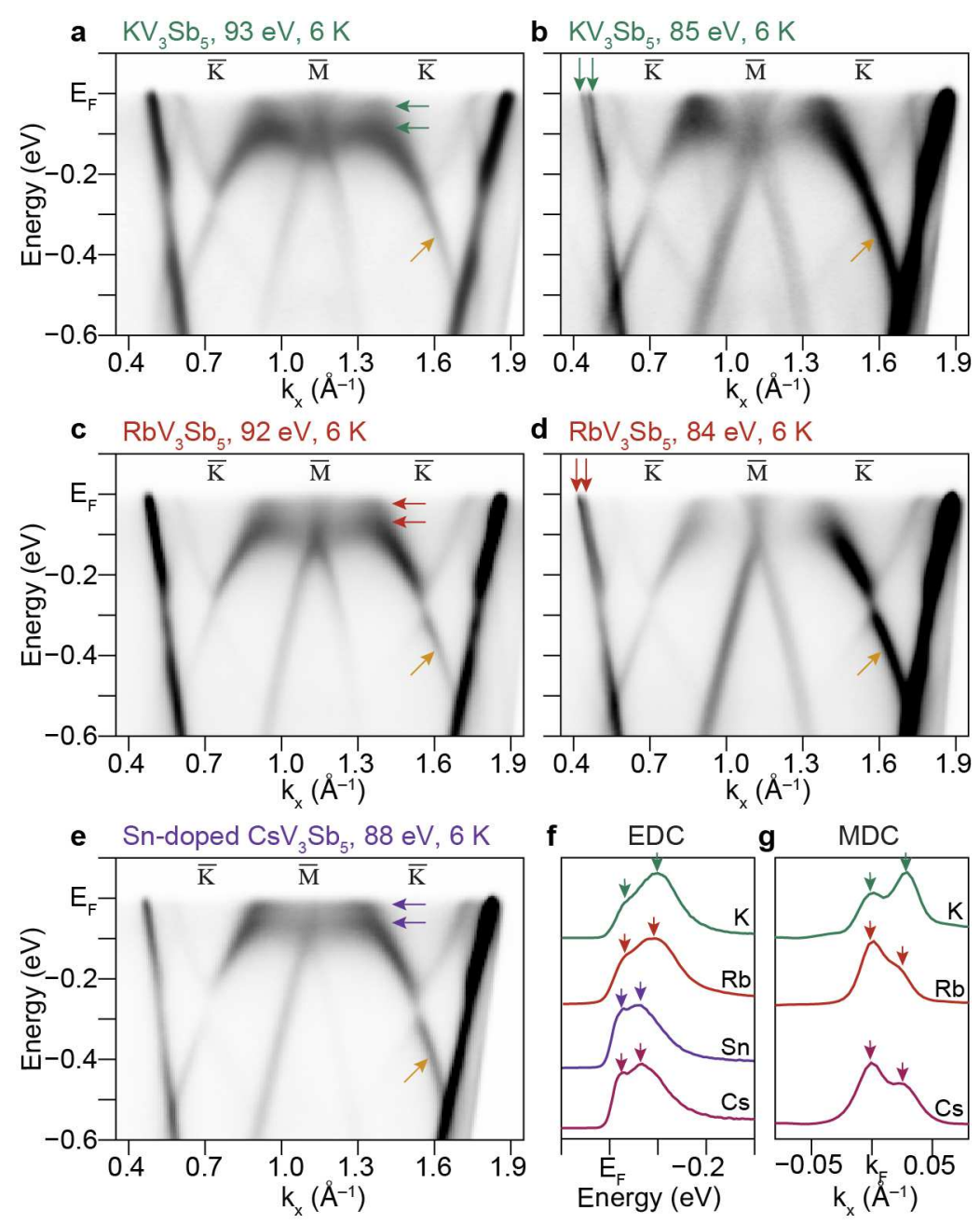

Figure 4 | Electronic reconstructions in $\mathrm{KV}_{3} \mathrm{Sb}_{5}, \mathrm{RbV}_{3} \mathrm{Sb}_{5}$, and $\mathrm{Sn}-\mathrm{doped} \mathrm{CsV}_{3} \mathrm{Sb}_{5}$ in 3D-CO state. a-e, ARPES spectra of $\mathrm{KV}_{3} \mathrm{Sb}_{5}(\mathrm{a}, \mathrm{b}) \mathrm{RbV}_{3} \mathrm{Sb}_{5}(\mathrm{c}, \mathrm{d})$, and $\mathrm{Sn}$-doped $\mathrm{CsV}_{3} \mathrm{Sb}_{5}$ (e) measured at $3996 \mathrm{~K}$. The spectra in a-e are collected with $93 \mathrm{eV}, 85 \mathrm{eV}, 92 \mathrm{eV}, 84 \mathrm{eV}$, and $88 \mathrm{eV}$ photons, 400 respectively. Green, red, and purple arrows indicate the splitting of $K 1$-vHS and K2-band. 401 Corresponding energy-distribution-curves (EDCs) and momentum-distribution curves (MDCs) 402 are shown in f,g (see below). Yellow arrows highlight the absence of splitting on the lower Dirac 403 band of $K 1$, which is markedly different from the case of $\mathrm{CsV}_{3} \mathrm{Sb}_{5}$ in Fig. 2g,i. f, EDCs measured 404 near the Fermi momentum $\left(\mathrm{k}_{\mathrm{F}}\right)$ of the K1-band. Arrows highlight the splitting of K1-band near 405 vHS. g, MDCs of the K2-band measured at the Fermi energy $\left(\mathrm{E}_{\mathrm{F}}\right)$. Arrows highlight the splitting 406 of the K2-band. 


\section{Extended Data Figures 1-3}
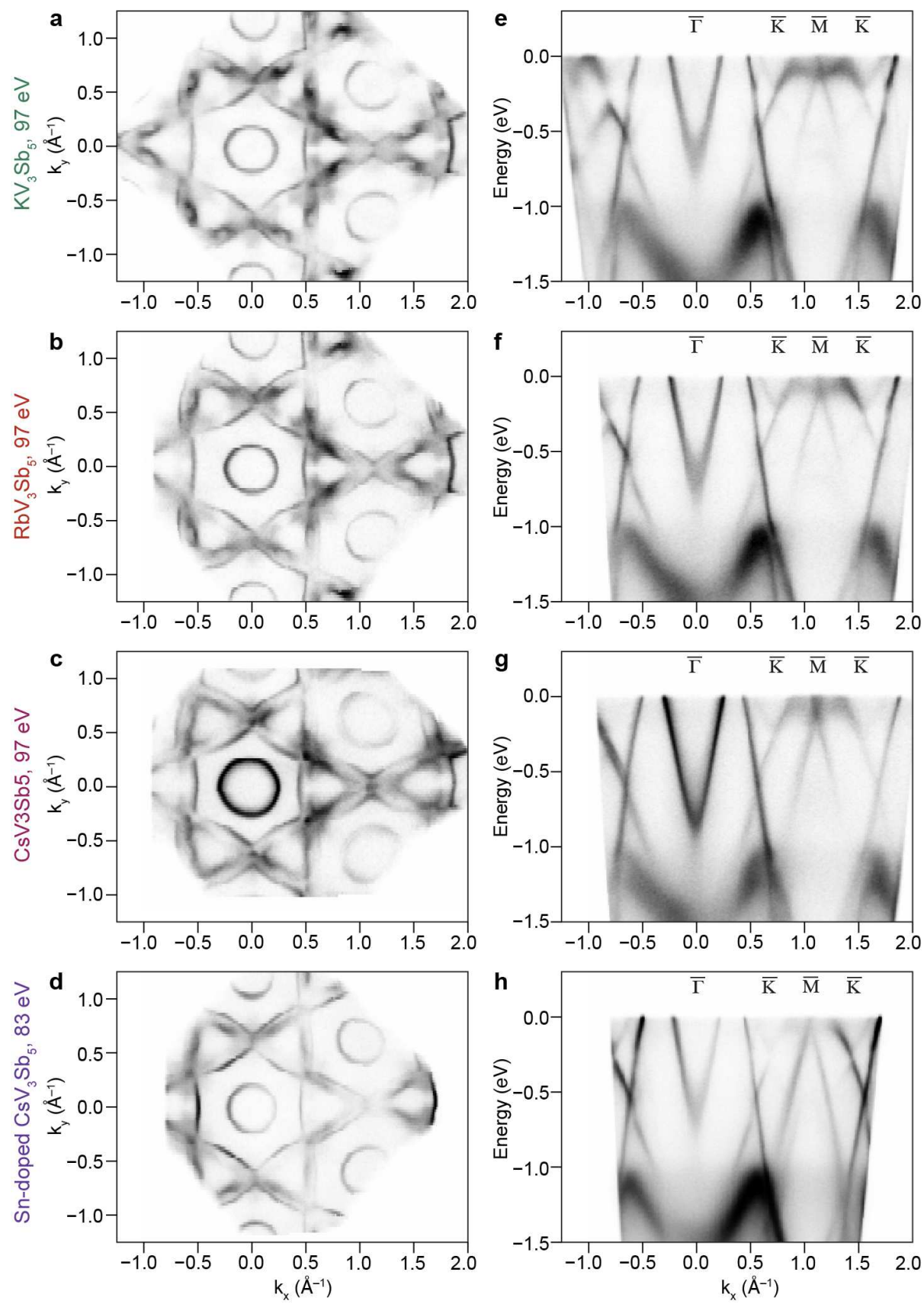

Extended Data Fig. 1 | Fermi surface and overall electronic structure of pristine and Sn-

$409 \mathrm{CsV}_{3} \mathrm{Sb}_{5}$, respectively. e-h, Wide energy-momentum range ARPES spectra of $\mathrm{KV}_{3} \mathrm{Sb}_{5}, \mathrm{RbV}_{3} \mathrm{Sb}_{5}$, $410 \mathrm{CsV}_{3} \mathrm{Sb}_{5}$ and $\mathrm{Sn}$-doped $\mathrm{CsV}_{3} \mathrm{Sb}_{5}$, respectively. All data are acquired at the base temperature $6 \mathrm{~K}$, 411 i.e. in the charge ordered state. 

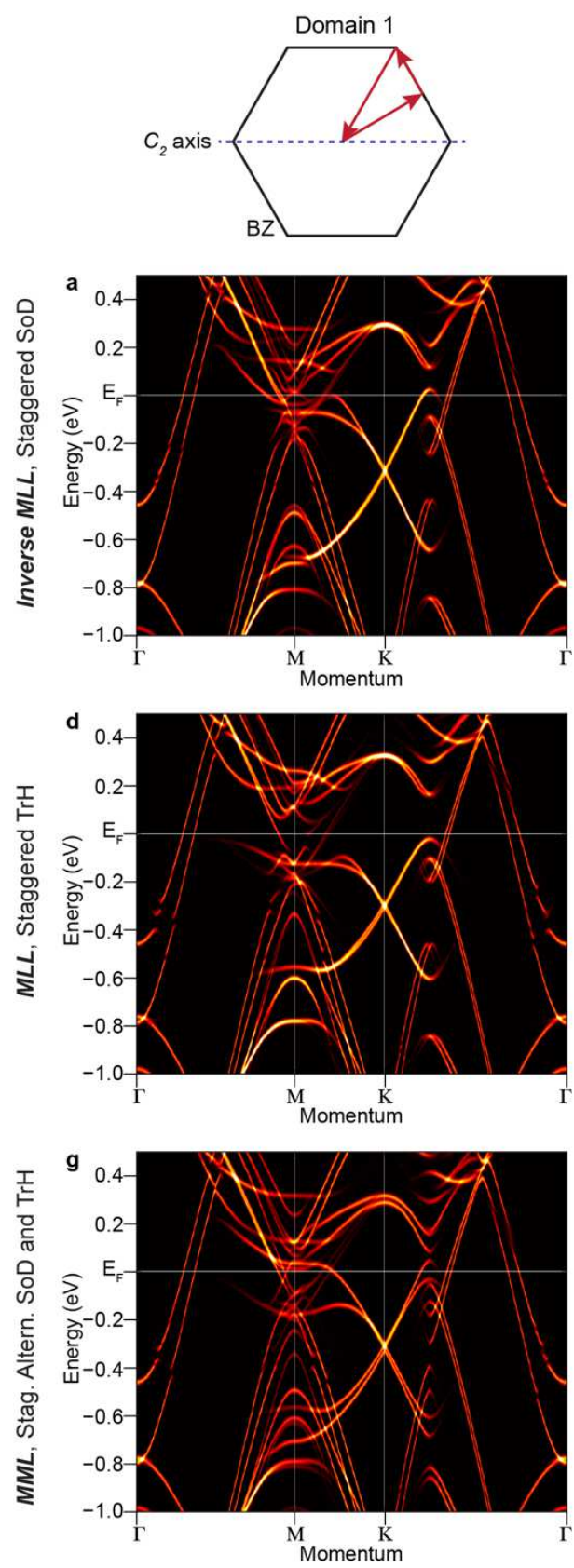
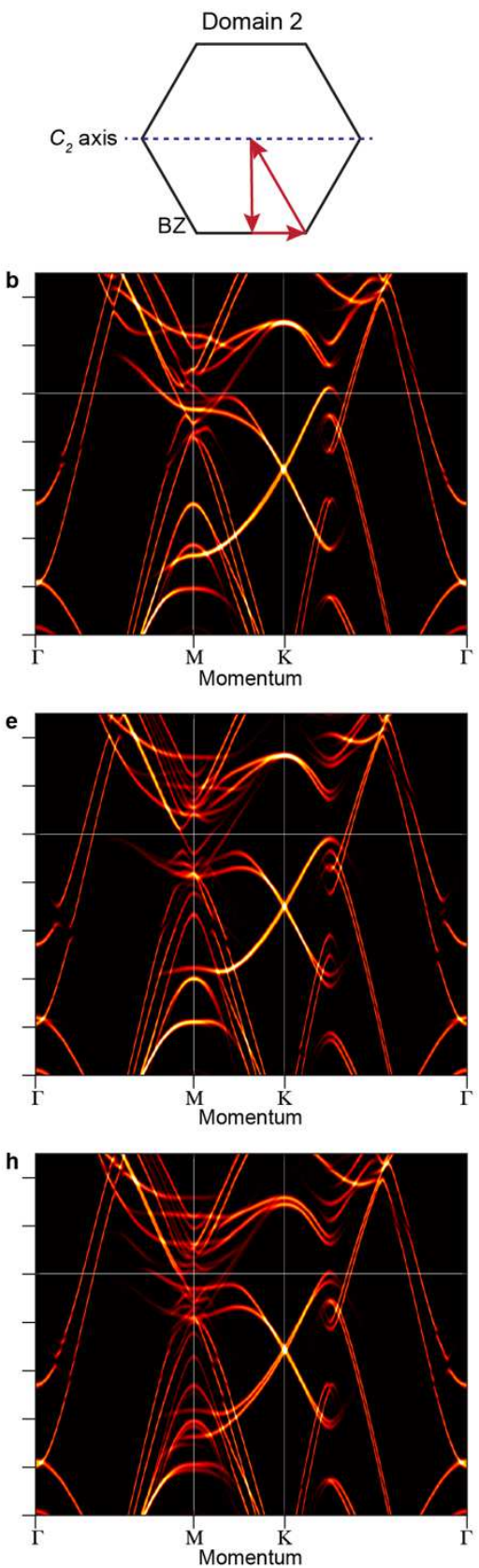
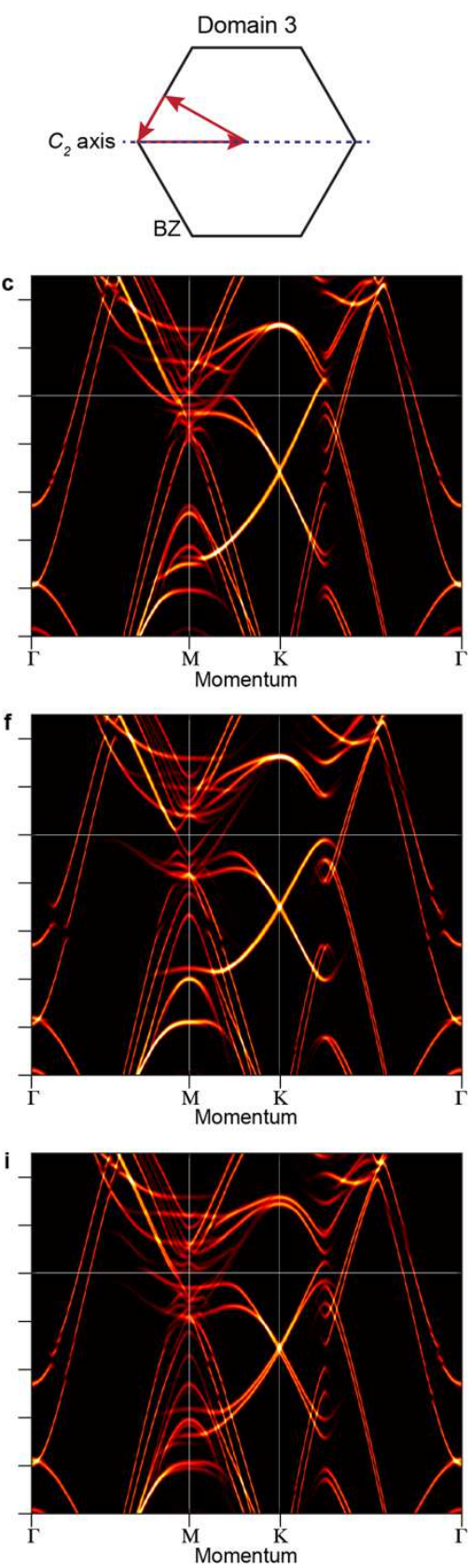

412 Extended Data Fig. 2 | Domain-resolved electronic structures of $\mathrm{CsV}_{3} \mathrm{Sb}_{5}$ in $C_{2}$ symmetric

413 3D-CO phases. a-c, Domain-resolved dispersion of $\mathrm{CsV}_{3} \mathrm{Sb}_{5}$ in the inverse $M L L 3 \mathrm{D}-\mathrm{CO}$ phase.

414 The location of three different $k$-paths with respect to the $C_{2}$ rotation axis is schematically

415 displayed above each panel. d-f, Same with a-c but in the $M L L$ phase. g-i, Same with a-c but in

416 the $M M L$ phase. Corresponding domain-averaged electronic structures of the inverse $M L L, M L L$,

417 and $M M L$ phases are shown in Fig. 3d-f. 

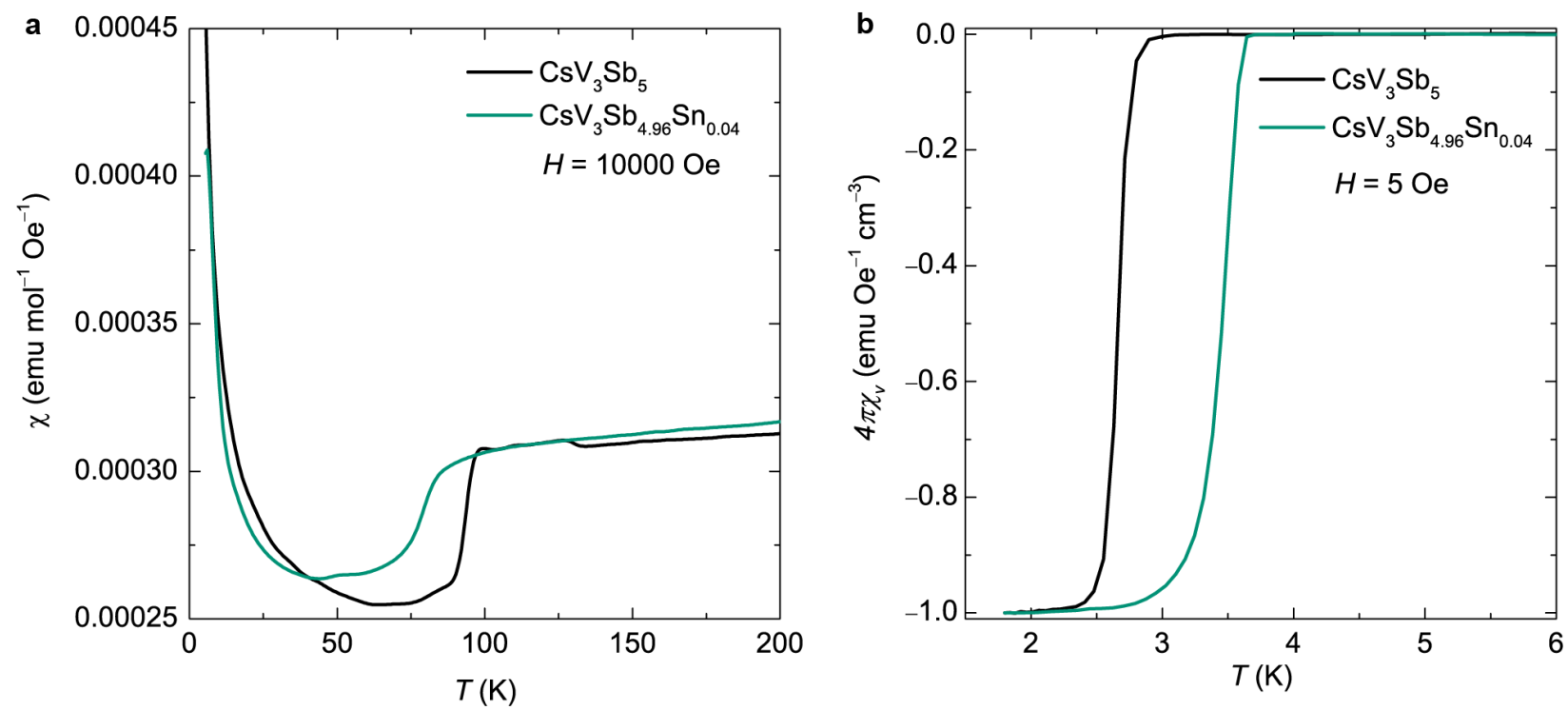

418 Extended Data Fig. 3 | Characterization of the Sn-doped $\mathrm{CsV}_{3} \mathrm{Sb}_{5}$ single crystal. a, Magnetic 419 susceptibility of the pristine and $\mathrm{Sn}$-doped $\mathrm{CsV}_{3} \mathrm{Sb}_{5}$ measured under a field of $H=10000 \mathrm{Oe}$. Sn 420 concentration (0.04 per unit cell) is determined by energy-dispersive X-ray spectroscopy. The 421 sharp drop in the magnetic susceptibility characterizes the onset of the charge order. With Sn422 doping, the $T_{\mathrm{CO}}$ decreases from $94 \mathrm{~K}$ to $79 \mathrm{~K}$. b, Low-temperature magnetic susceptibility 423 measured under a field of $H=5$ Oe. The perfect diamagnetic response $\left(4 \pi \chi_{\mathrm{v}}=-1\right)$ characterizes 424 the superconducting state. With Sn-doping, the superconducting transition temperature $T_{\mathrm{C}}$ 425 increases from $2.7 \mathrm{~K}$ to $3.4 \mathrm{~K}$ (estimated from the midpoint of transition), indicating a competition 426 between superconductivity and charge order in Sn-doped $A \mathrm{~V}_{3} \mathrm{Sb}_{5}$ series. $^{42}$ 